Bei dem erwähnten Glühlampenphotometer, dessen wesentliche Teile in Fig. 4 dargestellt sind, wird der Rahmen a (Fig. 3), der die Farbfilter enthält, mit Hilfe einer Schraubenspindel verschoben, die einen mit dem Zeiger $e$ versehenen Drehknopf $a$ trägt. Die Steigung der Schraube ist so bemessen, daß eine volle Drehung ausreicht, um den Rahmen von der einen in die andere Grenzstellung zu bewegen. Infolgedessen kam die Rahmenverschiebung direkt an der Skala $f$ abgelesen werden.

Von der aus Zinkblech hergestellten, innen mattweiB gestrichenen. Hohlkugel von $1.5 \mathrm{~m}$ Durchmesser, in deren Inneres die zu messende Lampe gebracht wird, ist in Fig. 4 nur ein Teil

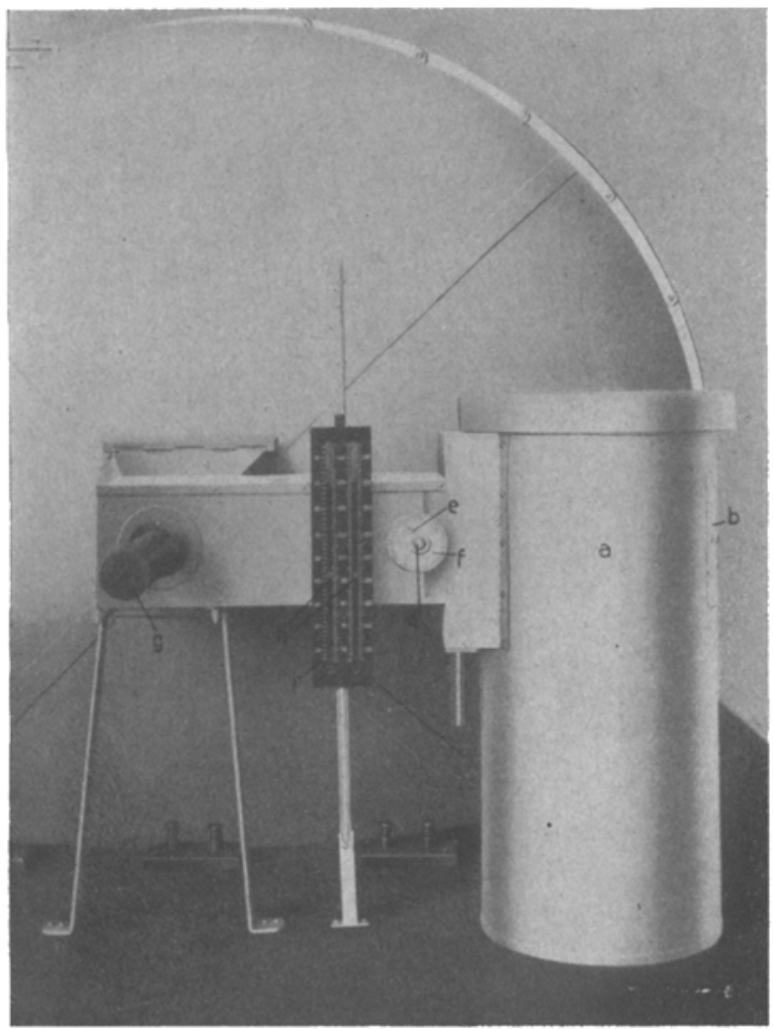

Fig. 4. Kugelphotometer für Betriebsmessungen an elektrischen Glühlampen.

sichtbar. Die Vergleichslampe, eine gewöhnliche Wolfram-Drahtlampe, befindet sich im Inneren des zylindrischen Behälters $a$ und beleuchtet zunächst eine Mattscheibe, die in dem sich auf der linken Seite anschließenden viereckigen Kasten angebracht ist. Das von dieser Mattscheibe ausgehende und durch die verschiebbaren Filter in der Farbe veränderliche licht wird nun in dem sich anschließenden länglichen, innen ebenfalls weiB gestrichenen Tubus weitergeleitet zu einer Mattscheibe, deren Helligkeit durch eine verstellbare Blende regelbar ist. Die Messung erfolgt nun in der Weise, daß man diese Blende so weit verstellt, bis die Helligkeit der von der Vergleichs- lampe erlevehteten Mattscheibe gleich der Helligkeit derjenigen wird, die das Kugelinnere abschliebt und somit von der zu messenden Lampe Licht erhät. Die Einstellung auf gleiche Helligkeit erfolgt mittels eines Lummer-Brodhunschen Würfels, der durch das Okular $g$ beobachtet wird. Die regelbare Blende, die durch einen in der Abbildung nicht sichtbaren Drehknopf betätigt wird, ist mit einem Doppelzeiger $h$ versehen, der auf der Skala $i$ spielt, an der man somit bei entsprechender Eichung die mittlere räumliche Lichtstärke der zu messenden Lampe unmittelbar ablesen kann.

Die größten Farbenunterschiede, die bei den praktisch verwendbaren elektrischen Glühlampen vorkommen, sind gegeben einerseits durch eine mit wesentlich verringerter Spannung, also entsprechend niedriger Temperatur brennenden Kohlefadeniampe und andererseits durch eine mit erhitzter Spannung betriebene sogenannte Halbwattlampe. Die Filter sind nun so abgestimmt, dab durch Drehung des Knopfes $d$ in wenigen Sekunden in diesen Grenzen auf jede beliebige Liehtfarbe eingestellt werden kann. Um die Temperatur des Giühfadens oder die ,Okonomie" an der Skala $f$ ablesen zu können, mub diese für eine bestimmte Lampenart vorher empirisch geeicht werden.

\section{Zur Beteiligung deutscher Gelehrter an der Ausbildung von Kampfmitteln.}

In den Zeitungen der Entente und selbst auch in der neutralen Presse sind wiederholt Angriffe gegen deutsche Gelehrte erhohen worden, weil sie die kriegerischen Maßnalimen ihrer Regierung unterstutzt und insbesondere auch an, wie behauptet wird, völkerrechtswidrigen Maßnalmmen dureh ihre Beihilfe sich indirekt beteiligt hätten, letzteres speziell, was die Ausbildung des sogenannten Gaskampfes betrifft.

Durch einseitige Information in der feindlichen und neutralen Presse sind hierubber im neutralen Ausland vielfach unrichtige Anffassungen entistanden. Die Gelehrten hatten, wie jeder andere Bürger, naturgemä $\beta$ gleichfalls die Verpflichtung, ihrem Vaterlande zu belfen und all ihr Können; wie geschehen, selbstlos einzusetzen.

\section{Allgemeines itber den Gaskampf.}

Der Umstand, daß die deutsehen Chemiker besonders wegen ihrer Beteiligung bei der Ausbildung das Gaskampfes angegriffen werden, ist psychologisch leieht zu verstehen. Es ist eine allgemeine Erfahrung, daß jedes neve Mittel der Kriegführung infolge der besonderen Bigenarten, die es mit sich. bringt, auch als eine neue Grausamkeit empfunden wird. An die schreckichen Verwundungen, die die modernen Sprenggranaten hervorrufen, war man gewöhnt; die Leiden ider Gaskranken, obwohl im allgemeinen nicht damit zu vergleichen, wurden als eine neue Begleiterscheinung des Irieges als grausam und unmenschlich hingestellt.

"Vom humanen Standpunkt ist es nicht grausamer, die Fieinde mit giftigen Gasen zu ersticken als sie im Wasser mit Hilfe von Torpedos zu ersäufen; jedes neue Kriegsmittel hat man immer zunächst als barbarisch bezeichnet und schließlich all gemein angenommen." 
Die vorstehenden Bemerkungen werden bei den Gegnern des Gaskampfes lebhaften Protest hervorrufen, und es sei daher gleich hinzugefïgt, daß sie nicht etwa vom Autor dieses Artikels, sondern von dem Vertreter der amerikanischen Regierung auf der Haager internationalen Konferenz im Jahre 1899, dem Admiral Mahan, stammen, wie denn bis auf den heutigen Tag die Vereinigten Staaten unter keinerlei Bestimmungen ihre Untensehrift gesetzt haben, die den Gaskampf einzuschränken oder zu verbieten geeignet waren.

Zweifellos kann man sagen, wäre 1914 der Gaskampi ein allgemein benutztes Kampfmittel gewesen, und hätten die deutschen Gelehrten während des Krieges neben den Gasgranaten die Lydditsprenggeschosse eingeführt, so wurde die Entrüstung uber diese Schandtat bei der Entente und bei so manchem Neutralen noch ungleich größer gewesen sein.

\section{Vorgeschichte des Gaskampfes.}

Ubrigens ist der Gaskmmpt keinoswegs eine so neue bösartige Erfindung, wie die feindliche Presse fortwährend behauptet hat. Eingehende, aber technisch unvollkommene Vorschläge, um den durch Bollwerke verdeckten Feind mit Hilfe von Giftschwaden (Arsenikdämpten und dergleichen) unschädlich zu machen, beschreibt bekanntlich bereits Lionardo da Vinci, ohne daß man ihn deshalb zu einem Auswur $\tilde{f}$ der Menschheit gestempelt hätte. Einen eingehend durchgearbeiteten Vorechlag") hat wihrend des Krimkrieges der Admiral Lord Graf von Dundonald, einer der berïhmtesten englischen Seehelden, aufgestellt, dahingehend, Sebastopol mit Hilfe einer Gaswolke, erzeugt durch verbrannten Schwefel, zu nehmen. Dieser Plan wurde nicht etwa a limine abgewiesen, sondern sorgfältig erwogen, schlieblich aber abgelehnt, wobei es dahin gestellt bleiben mag, ob humanitäre Rücksichten bei der Ablehnung des, wie "wir heute wissen, technisch recht unvollkommenen Projektes eine maBgebende Rolle gespielt haben.

Diejenige Macht, die sich zuerst erfolgreich mit der Ausbildung eines Gaskampfimittels beschäftigt hat, ist, soweit bis jetzt bekannt, Frankreich gewesen; bereits vor dem Kriege trat nämlich in der französischen Armee eine sogenannte Gewrehrgasgranate in Aktion, die bej dem wohlbekannten Angrift auf die Bonnetsche Apachenbande in Choisy-le-Roy benutzt wurde; dies uns heute wohlbekannte Geschob enthielt Bromessigwther, eine Müssigkeit, die in feinverteilter Form zerstäubt durch die stark tränenerregende und die Atmungsorgane stark ätzende Wirkung den Aufenthalt in Schützengräben und Unterständen unmöglich macht. Es steht auch fest, dab diese Waffe, ebenso wie eine ahnlich konstruierte Handgranate, bereits Anfang 1915, wenn nicht früher, gegen das deutsche Heer zur Verwendung gelangte. Da aber damals den Franzosen offenbar nur sehr beschränkte Mengen des erwähnten Präparates zur Verfugung standen, so war der praktische Erfolg gering und die offentlichkeit hat davon bisher kanm erfahren. Auf die Bemühungen des bekannten Sprengstofftechnikers Turpin betreffis der Verwendung von Giftgasen, die gleichfalls por die Zeit von 1914 fallen, wird weiter unten noch hingewiesen werden.

Jedenfalls lehren die in diesem Abschnitte mitgeteilten Tatsachen, die sich noch nach vielen Richtun-

1) Vgl. hieruber den interessanten Artikel von $O l a$ rence $J$. West (Chemical Warfare Service) in der ,Science rom $^{\text {2. Mai }} 1919$. gen erweitern ließen, daß Gaskampimittel bereits vor dem Kriege vielfach bearbeitet worden sind.

\section{Gaskampf und Vollerrecht.}

Gegen diejenigen, die in Deutschland an der Ausbildung des Gaskampfes mitgewirkt haben - von einer Erfindung des Gaskampfes in Deutschland kann nach den vorstehenden Mitteilungen nicht mehr gesprochen werden -, wind vielfach der Vorwurf erhoben, dab sie durch ihre Mitarbeit sich einer Verletzung des Volkerrechts schuldig gemacht hätten.

Allgemein ist hierzu zu bemerken, daß die Aufgabe des Gelehrten völlig zu trennen ist von der Verwendung der durch seine wissenschaftliche Forsehung enzielten Ergebnisse und von der Frage, ob diese Verwendung völkerrechtlichen Grundsätzen entspricht.

Es würde deshalb den Rahmen dieses Artikels weit iiberschreiten, wenn die völkerrechtliche Frage hier eingehend erörtert oder gar entschieden werden sollte. Aber folgende Tatsachen seien hervorgehoben. Auf der Haager Konferenz vom Jahre 1899 war beantragt, Geschosse zu verbieten, deren einziger Zweek ist, „erstickende oder giftige Gase zu verbreiten". England und Nordamerika widersprachen damals, erst auf der Konferenz von 1907 gab England nach, während die Vereinigten Staaten auf ihrem Widerspruche beharrten.

Ubrigens ist die erwähnte Klausel offenbar mit Absicht so unbestimmt gefaßt, daß ernistliche Zweifel im gegebenen Falle auftreten, ob ein Kampimittel darunter fallt oder nicht. Im Grunde genommen ist nämlich jede moderne Granate zugleich ein unter Umständen sehr wirksames Gasgeschoß; die Füllung der Granate (Pikrinsänure, Trinitrotoluol oder dergieichen) liefert bei der Detonation sehr erhebliche Mengen von Kohlenoxyd und auch Stickoxyd, zwei Gasen von starker Giftwirkung. Die Zahl der dadureh veranlaßten Todesfälle, besonders in geschlossenen Räumen, ist während des Krieges gewaltig groß gewesen. Da es nun nicht in der Absicht der Haager Konferenz lag, in Zukuntt diese Gaswirkungen und damit die stärksten Sprengstoffe auszuschließen, so war eine sachgemäße juristische Formulierung des Verbotes von giftige Gase verbreitenden Geschossen von vornherein eine kaum losbare Aufgabe.

Geschosse, die nach dem Muster der französischen Gewehrgranaten konstruiert und wegen ihrer Größe ungleich wirksamer gewesen sind, wurden ungefähr gleichzeitig von der deutschen Kriegführung in groBem Umfange und mit unleugbarem Erfolge verwendet. Dieselben enthielten aber im Gegensatz zu der erwähnten französischen Gewehrgranate stets eine sehr beträcht. liche Sprengladung und übten daher gleichzeitig eine starke Splitterwirkung aus, können also unmöglich als Geschosse bezeichnet werden, ,deren einziger Zweck es ist, erstickende oder giftige Gase zu verbreiten". Erst im Frühjahr 1916 wurden von französischer Seite Geschosse verwendet, die eine so schwache Sprengiadung besaßen, daß sie von unserer Truppe von Blindgängern nicht unterschieden werden konnten.

Die Haager Konferenz hat ferner die Anwendung van Giften und giftigen Waffen verboten. Aus dem ganzen Zusammenhang ist klar, daß es sich hier um Brunnenvergiftungen und derartiges handelt, nieht um das Ausränchern des Feindes mit Stinktöpfen, Feuerwerksätzen und dergleichen, wie es früber üblich war und wie es in diesem Kriege in Gestait der Chlorwolken in technisch stark vergröbertem und daher ungleich wirksamerem MaBstabe zuerst von deutseher 
Seite, später auch vom Gegner in Anwendung gebracht ist.

\section{Ausbildung des deutschen Gaskampfes}

Bei Beginn des Weltkrieges hatte Deutschland im Gegensatz zu Frankreich keinerlej Gaskampfmittel ausgebildet; die Gefahr lag nahe, daß Frankreich den gewonnenen Vorsprung ausnützen werde; ein am 28. September 1914 in der italienischen Zeitung" "La Tribuna" ersehienener Aufsatz, wonach Turpin Gasbomben von nnerkört weitgehender, tödlicher Wirkung erfunden haben solle, dürfte die leitenden militärischen Stellen in dieser Auffasisung bestärkt haben. Hervorzuheben ist ubrigens, das weder die "Tribuna" noch eine englische oder französische Zeitung vor der Benutzung der Turpinschen Erfindung, die vermutlich auf der Verwendung des höchst giftigen stickoxydes beruhte, gewarnt hat. Jedenfalls war die deutsche Oberste Heeresleitung und das deutsche Kriegsministerium auf Grund der erwähnten drohenden Anzeichen verpflichtet, den Gaskampimitteln ihre volle Aufmerksam. keit zuzuwenden, und als in diesem Sinne eine Aufforderung an deutsche Chemiker erging, waren diese natürlich nicht minder gehalten, in dem angeregten Sinne zu arbeiten; hätten sie unter Berufung auf ein vermeintliches Verbot des Völkerrechts abgelehnt, so häten sie sich mit Recht dem Vorwurf ausgesetat, aus Gründen, die sie nichts angehen und die sie nicht beurteilen können, sich ihrer patriotischen Pflicht entzogen zu haben. Hinzu kommt, daß nach den Bestimmungen der Haager Konvention die Beschränkungen inmer unter der Voraussetzung der Gegenseitigkeit in Wirksamkeit sind. Kam durch eine gewiß nicht deutschfreundliche Tageszeitung die unwidersprochene Nachricht, daß die Gegner unter Führung eines bekannten Fachmannes den Gaskampf in großem Stile vorbereiten, so muBte die deutsche Wissenschaft alles tun, um ihrerseits die $A b w e h r$ dieses Angriffes vorzubereiten. Sie konnte mit ibren Versuchen nicht warten, bis die Richtigkeit der Nachrichten durch Anwendung der Mittel bestätigt wurde; wer angesichts dieser Sachlage seine Kraft dern Vaterlande zur Verŝugung stellte, kann niemals unmoralisch gehandelt haben, gleichgultig in welcher Form das Heer von den Ergebnisen seiner Forschung Gebranch machte und welche Folgen daraus entstanden sind. Unsere Gegner zögerten hierauf nicht, auch ihrenseits nunmehr den Gaskampf in größtem Maßstabe zu organisieren.

\section{Die Zukunft des Gaskrieges.}

Wenn wirklich Deutschland auf dem Gebiete des Gaskampies sich an der Menschlichkeit versündigt haben sollte, so müBte man doch erwarten, daB nunmehr alle zivilisierten Völker einstimmig in der $A b-$ schaffung der Gaskampfmittel sein müBten. Davon ist nun gar keine Rede. Es ist schon oben betont, daß Nordamerika in der Verwendung giftiger Gase nie sich eine Beschränkung hat auferlegen lassen. Aber zahilreiche Anzeichen deuten darauf hin, daß auch die anderen kriegführenden Mächte keineswegs gesonnen sind, in Zukunft die Gasgesehosse aus der Reihe ihrer Kampfmittel $z u$ streichen. Sehr charakteristisch sind die Ausfïhrungen des englischen Professors A. Snithells ${ }^{1}$ ) von der Universität Leeds, eines Forschers, der, wenn wir recht berichtet sind, an der Ausarbeitung des Gaskampfes sich eingehend be-

1) Vgl. Zeitschr. f. angewandte Chemie Nr. 41 vom 23. Mai 1919, S. 331 . teiligt hat; Smithells äuBert sich nämlich dahin, daB, wenn es wieder Kriege geben wärde, höchstwahrsehein. lich auch der Gaskampf wieder eine Rolle spielen würde. „Man habe die Verwendung von Giftgasen als Kampfmittel grausam und unnatirlich genannt, unzweifelhaft sei dies auch im Anfang so gewesen. Aber man milsse bedenken, daß man jede neue Methode der Kriegfïhrung, auch die Einführung des Schießpulvers, als grausam bezeichnet habe. Nachdem man aber einmal mit diesem Kriegsmittel bekannt geworden sei, habe es seine Sehrecken verloren." Frr erklärt es ferner für unmöglich, daß England jemals in Zukunft auf die Verwendung von Giftgasen werde verzichten können.

Mit anderen Worten, die technische Entwicklung der Kriegskunst ist genau den Weg gegangen, den Admiral Mahan (vergl. oben $\mathrm{S}$. 793) mit prophetischem Blicke vorausgesagt hatte. Nicht eine einzelne Nation oder gar eine einzelne Persönlichkeit, sondern die lange Dauer des Weltkrieges und der Umstand, daß der Stellungskrieg zu früher ungeahnter Vollendung ausgebildet wurde, trägt die Schuld an dieser Entwicklung. Dem Philantropen bleibt nur die Hoffnung abrig, daB, nachdem die so zahlreichen Schrecken eines großen modernen Krieges dureh die Ausbildung der Gaswaffen um einen neuen vermehrt sind und nachdem andererseits durch die in Zukunft notwendige Beschaffung, der Gasschutzmittel die Vorbereitungen für einen neuen Kampf, eine weitere Erschwerung er. fahren haben, die Menschheit es sich in Zukunft um so mehr überlegen wird, die furchtbaren Folgen eines neuen Riesenkampfes auf sich zu nehmen.

\section{A. Berliner, Berlin.}

\section{Besprechungen.}

Uibe, Martin, Über die Helligkeitsverteilung des diffusen Sonnenlichts am klaren Himmel. Abhandl. der math.-phys. Klasse der Sächsischen Gesellschaft der Wiss. 35, VI, 319-367. Leipzig; B. G. Teubner, 1918. Preis M. 2,40.

Die Helligkeitsverteilung des diffusen Sonnenlichtes am klaren Himmel verdient wegen ihrer engen Beziehung zu den atmosphärischen Polarisationserscheinungen, wegen ihrer Bedeutung tür gewisse aktuelle Aufgaben der Astronomie usw. ein besonderes Interesse. Es ist daher sehr zu begrüBen, daß Herr Martin Uibe, Mitglied der Teneriffaexpedition unter Leitung Dombers, sie einer erneuten Untersuchnig unterzogen hat, deren Wert besonders darin besteht, daß sie unter den hervorragend günstigen kllimatischen und geographischen Verhältnissen von Teneriffa ausgefuhrt wurde. Beobachtet wurde im Sommer 1914, abgesehen von einigen Vorbereitungsmessungen auf der Alta Vista, auf der äupersten Spitze des Pico de Teide $(3700 \mathrm{~m})$, und im Sommer 1916 in dem städtchen Guimar $(300 \mathrm{~m})$. Die maximale Sonnenböhe beträgt für Teneriffa $85^{\circ}$, es konnten also die Erscheinungen für praktisch alle Sonnenhöhen untersucht werden. Die Messungen wurden im unzerlegten Licht mittels eines 0 . Wienerschen Photometers zur Bestimmung der Himmelshelligkeit ausgeführt, in dem die ursprïnglich vorgesehenen Meskeile durch ein FlüssigkeitsgefäB mit meBbar veränderlicher Dicke der vom Licht durchlaufenen Flüssigkeits. sehicht ersetzt waren. Die ursprüngliche Form des Instrumentes ist in der Zeitschrift für Instrumentenkande 22, 55, 1912 von Herxheimer beschrieben. Die benutzte Flïssigkeit war eine neutralgraue Lösung von 